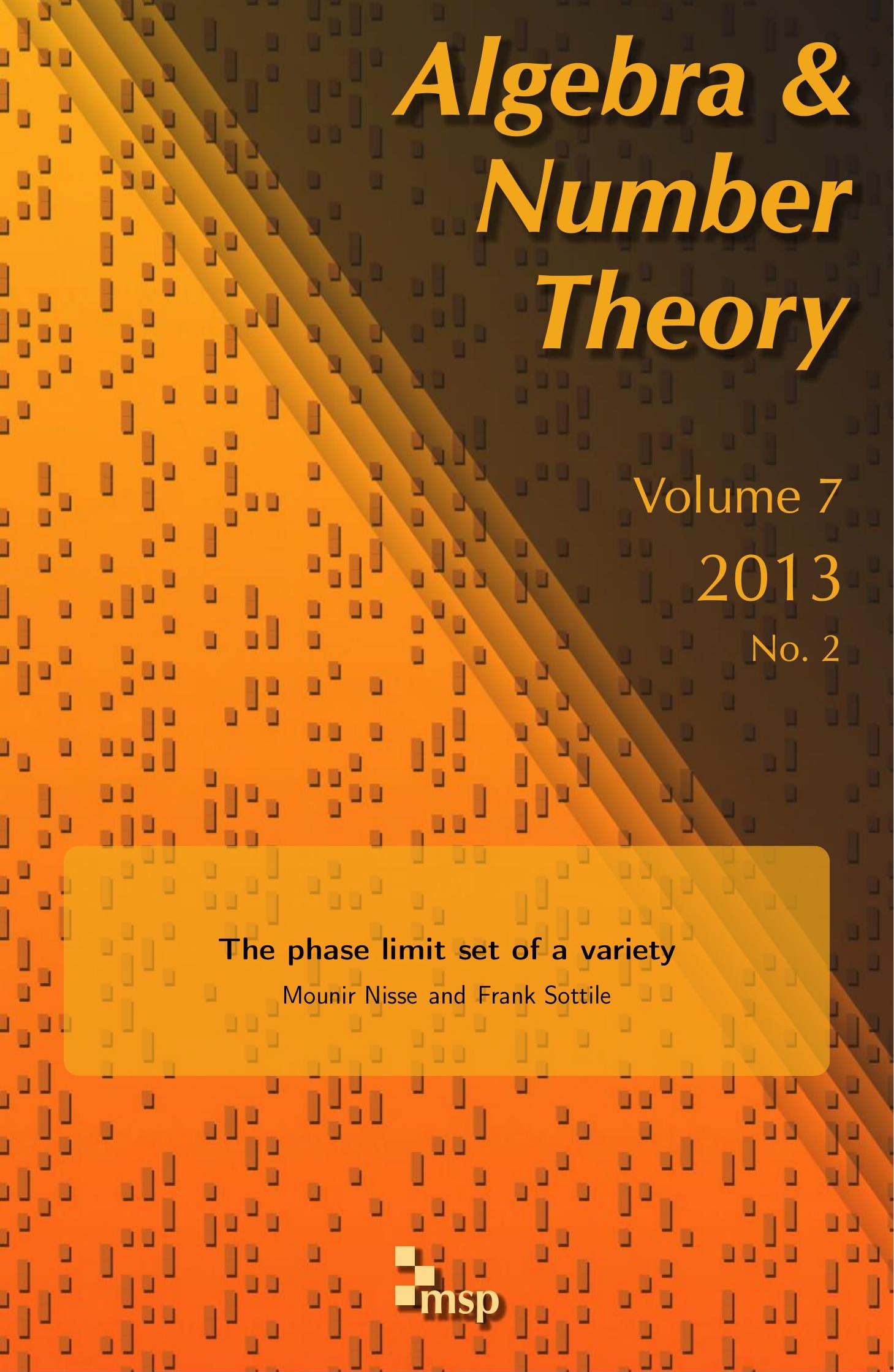




\title{
The phase limit set of a variety
}

\author{
Mounir Nisse and Frank Sottile
}

\begin{abstract}
A coamoeba is the image of a subvariety of a complex torus under the argument map to the real torus. We describe the structure of the boundary of the coamoeba of a variety, which we relate to its logarithmic limit set. Detailed examples of lines in three-dimensional space illustrate and motivate these results.
\end{abstract}

\section{Introduction}

A coamoeba is the image of a subvariety of a complex torus under the argument map to the real torus. Coamoebae are cousins to amoebae, which are images of subvarieties under the coordinatewise logarithm map $z \mapsto \log |z|$. Amoebae were introduced by Gelfand, Kapranov, and Zelevinsky [1994] and have subsequently been widely studied [Kenyon et al. 2006; Mikhalkin 2000; Passare and Rullgård 2004; Purbhoo 2008]. Coamoebae were introduced by Passare in a talk in 2004, and they appear to have many beautiful and interesting properties. For example, coamoebae of $\mathscr{A}$-discriminants in dimension 2 are unions of two nonconvex polyhedra [Nilsson and Passare 2010], and a hypersurface coamoeba has an associated arrangement of codimension-1 tori contained in its closure [Nisse 2009].

Bergman [1971] introduced the logarithmic limit set $\mathscr{L}^{\infty}(X)$ of a subvariety $X$ of the torus as the set of limiting directions of points in its amoeba. Bieri and Groves [1984] showed that $\mathscr{L}^{\infty}(X)$ is a rational polyhedral complex in the sphere. Logarithmic limit sets are now called tropical algebraic varieties [Speyer and Sturmfels 2004]. For a hypersurface $\mathscr{V}(f)$, logarithmic limit set $\mathscr{L}^{\infty}(\mathscr{V}(f))$ consists of the directions of nonmaximal cones in the outer normal fan of the Newton polytope of $f$. We introduce a similar object for coamoebae and establish a structure theorem for coamoebae similar to those of Bergman and of Bieri and Groves for amoebae.

Let $\operatorname{co} A(X)$ be the coamoeba of a subvariety $X$ of $\left(\mathbb{C}^{*}\right)^{n}$ with ideal $I$. The phase limit set $\mathscr{P}^{\infty}(X)$ of $X$ is the set of accumulation points of arguments of sequences in $X$ with unbounded logarithm. For $w \in \mathbb{R}^{n}$, the initial variety $\operatorname{in}_{w} X \subset\left(\mathbb{C}^{*}\right)^{n}$ is the variety of the initial ideal of $I$. The fundamental theorem of tropical geometry

MSC2010: primary 14T05; secondary 32A60.

Keywords: coamoeba, amoeba, initial ideal, toric variety tropical geometry. 
asserts that $\operatorname{in}_{w} X \neq \varnothing$ exactly when the direction of $-w$ lies in $\mathscr{L}^{\infty}(X)$. We establish its analog for coamoebae.

Theorem 1. The closure of $\operatorname{coA}$ is $\operatorname{coA}(X) \cup \mathscr{P}^{\infty}(X)$, and

$$
\mathscr{P}^{\infty}(X)=\bigcup_{w \neq 0} \operatorname{coA}\left(\operatorname{in}_{w} X\right)
$$

Johansson [2013] used different methods to prove this when $X$ is a complete intersection.

The cone over the logarithmic limit set admits the structure of a rational polyhedral fan $\Sigma$ in which all weights $w$ in the relative interior of a cone $\sigma \in \Sigma$ give the same initial scheme $\operatorname{in}_{w} X$. Thus, the union in Theorem 1 is finite and indexed by the images of these cones $\sigma$ in the logarithmic limit set of $X$. The logarithmic limit set or tropical algebraic variety is a combinatorial shadow of $X$ encoding many properties of $X$. While the coamoeba of $X$ is typically not purely combinatorial (see the examples of lines in $\left(\mathbb{C}^{*}\right)^{3}$ in Section 3 ), the phase limit set does provide a combinatorial skeleton and that we believe will be useful in the further study of coamoebae.

We give definitions and background in Section 2 and detailed examples of lines in three-dimensional space in Section 3. These examples are reminiscent of the concrete descriptions of amoebae of lines in [Theobald 2002]. We prove Theorem 1 in Section 4.

\section{Coamoebae, tropical varieties, and initial ideals}

As a real algebraic group, the set $\mathbb{T}:=\mathbb{C}^{*}$ of invertible complex numbers is isomorphic to $\mathbb{R} \times \mathbb{U}$ under the map $(r, \theta) \mapsto e^{r+\sqrt{-1} \theta}$. Here, $\mathbb{U}$ is the set of complex numbers of norm 1 that may be identified with $\mathbb{R} / 2 \pi \mathbb{Z}$. The inverse map is $z \mapsto(\log |z|, \arg (z))$.

Let $M$ be a free abelian group of finite rank and $N=\operatorname{Hom}(M, \mathbb{Z})$ its dual group. We use $\langle\cdot, \cdot\rangle$ for the pairing between $M$ and $N$. The group ring $\mathbb{C}[M]$ is the ring of Laurent polynomials with exponents in $M$. It is the coordinate ring of a torus $\mathbb{T}_{N}$ that is identified with $N \otimes_{\mathbb{Z}} \mathbb{T}=\operatorname{Hom}(M, \mathbb{T})$, the set of group homomorphisms $M \rightarrow \mathbb{T}$. There are natural maps Log: $\mathbb{T}_{N} \rightarrow \mathbb{R}_{N}=N \otimes_{\mathbb{Z}} \mathbb{R}$ and arg: $\mathbb{T}_{N} \rightarrow \mathbb{U}_{N}=N \otimes_{\mathbb{Z}} \mathbb{U}$, which are induced by the maps $\mathbb{C}^{*} \ni z \mapsto \log |z|$ and $z \mapsto \arg (z) \in \mathbb{U}$. Maps $N \rightarrow N^{\prime}$ of free abelian groups induce corresponding maps $\mathbb{T}_{N} \rightarrow \mathbb{T}_{N^{\prime}}$ of tori and also of $\mathbb{R}_{N}$ and $\mathbb{U}_{N}$. If $n$ is the rank of $N$, we may identify $N$ with $\mathbb{Z}^{n}$, which identifies $\mathbb{T}_{N}$ with $\mathbb{T}^{n}, \mathbb{U}_{N}$ with $\mathbb{U}^{n}$, and $\mathbb{R}_{N}$ with $\mathbb{R}^{n}$.

The amoeba $\mathscr{A}(X)$ of a subvariety $X \subset \mathbb{T}_{N}$ is its image under $\log : \mathbb{T}_{N} \rightarrow \mathbb{R}_{N}$, and the coamoeba $\operatorname{co} \mathscr{A}(X)$ of $X$ is the image of $X$ under $\arg : \mathbb{T}_{N} \rightarrow \mathbb{U}_{N}$. An amoeba has a geometric-combinatorial structure at infinity encoded by the logarithmic limit 
set [Bergman 1971; Bieri and Groves 1984]. Coamoebae similarly have phase limit sets that have a related combinatorial structure that we define and study in Section 4.

If we identify $\mathbb{C}^{*}$ with $\mathbb{R}^{2} \backslash\{(0,0)\}$, then the map arg: $\mathbb{C}^{*} \rightarrow \mathbb{U}$ given by $(a, b) \mapsto$ $(a, b) / \sqrt{a^{2}+b^{2}}$ is a real algebraic map. Thus, coamoebae, as they are the image of a real algebraic subset of the real algebraic variety $\mathbb{T}_{N}$ under the real algebraic map arg, are semialgebraic subsets of $\mathbb{U}_{N}$ [Basu et al. 2006]. It would be very interesting to study them as semialgebraic sets; in particular, what are the equations and inequalities satisfied by a coamoeba? When $X$ is a Grassmannian, such a description would generalize Richter-Gebert's five-point condition for phirotopes from rank 2 to arbitrary rank [Below et al. 2003].

Similarly, we may replace the map $\mathbb{C}^{*} \ni z \mapsto \log |z| \in \mathbb{R}$ in the definition of amoebae by the map $\mathbb{C}^{*} \ni z \mapsto|z| \in \mathbb{R}^{+}:=\{r \in \mathbb{R} \mid r>0\}$ to obtain the algebraic amoeba of $X$, which is a subset of $\mathbb{R}_{N}^{+}$. The algebraic amoeba is a semialgebraic subset of $\mathbb{R}_{N}^{+}$, and we also ask for its description as a semialgebraic set.

Example 2. Let $\ell \subset \mathbb{T}^{2}$ be defined by $x+y+1=0$. The coamoeba $\operatorname{co} A(\ell)$ is the set of points of $\mathbb{U}^{2}$ of the form $(\arg (x), \pi+\arg (x+1))$ for $x \in \mathbb{C} \backslash\{0,-1\}$. If $x$ is real, then these points are $( \pm \pi, 0),( \pm \pi, \pm \pi)$, and $(0, \pm \pi)$ if $x$ lies in the intervals $(-\infty,-1),(-1,0)$, and $(0, \infty)$, respectively. For other values, consider the picture below in the complex plane:

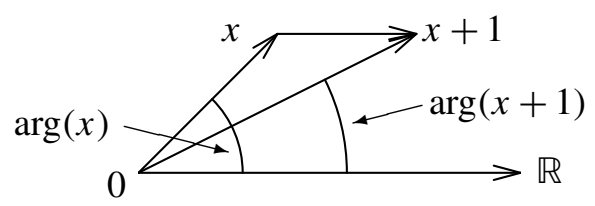

For $\arg (x) \notin\{0, \pi\}$ fixed, $\pi+\arg (x+1)$ can take on any value strictly between $\pi+\arg (x)$ (for $w$ near $\infty$ ) and 0 (for $x$ near 0 ), and thus, $\operatorname{co} A(\ell)$ consists of the three points $(\pi, 0),(\pi, \pi)$, and $(0, \pi)$ and the interiors of the two triangles displayed below in the fundamental domain $[-\pi, \pi]^{2} \subset \mathbb{R}^{2}$ of $\mathbb{U}^{2}$. This should be understood modulo $2 \pi$ so that $\pi=-\pi$.

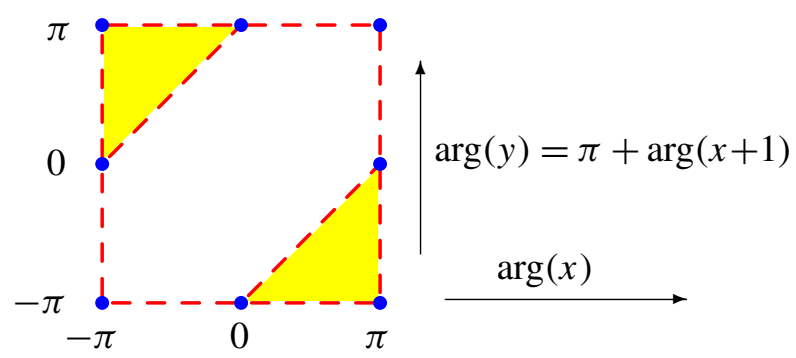

The coamoeba is the complement of the region

$$
\left\{(\alpha, \beta) \in[-\pi, \pi]^{2}|| \alpha-\beta \mid \leq \pi=\arg (-1)\right\}
$$


together with the three images of real points $( \pm \pi, 0),( \pm \pi, \pm \pi)$, and $(0, \pm \pi)$.

Given a general line $a x+b y+c=0$ with $a, b, c \in \mathbb{C}^{*}$, we may replace $x$ by $c x^{\prime} / a$ and $y$ by $c y^{\prime} / b$ to obtain the line $x^{\prime}+y^{\prime}+1=0$ with coamoeba (1). This transformation rotates the coamoeba (1) by $\arg (a / c)$ horizontally and $\arg (b / c)$ vertically.

Let $f \in \mathbb{C}[M]$ be a polynomial with support $\mathscr{A} \subset M$

$$
f:=\sum_{\boldsymbol{m} \in \mathscr{A}} c_{\boldsymbol{m}} \cdot \xi^{\boldsymbol{m}}, \quad \text { where } c_{\boldsymbol{m}} \in \mathbb{C}^{*},
$$

and we write $\xi^{\boldsymbol{m}}$ for the element of $\mathbb{C}[M]$ corresponding to $\boldsymbol{m} \in M$. Given $w \in \mathbb{R}_{N}$, let $w(f)$ be the minimum of $\langle\boldsymbol{m}, w\rangle$ for $\boldsymbol{m} \in \mathscr{A}$. Then the initial form $\operatorname{in}_{w} f$ of $f$ with respect to $w \in \mathbb{R}_{N}$ is the polynomial in $\operatorname{in}_{w} f \in \mathbb{C}[M]$ defined by

$$
\operatorname{in}_{w} f:=\sum_{\langle\boldsymbol{m}, w\rangle=w(f)} c_{\boldsymbol{m}} \cdot \xi^{\boldsymbol{m}} .
$$

Given an ideal $I \subset \mathbb{C}[M]$ and $w \in \mathbb{R}_{N}$, the initial ideal with respect to $w$ is

$$
\operatorname{in}_{w} I:=\left\langle\operatorname{in}_{w} f \mid f \in I\right\rangle \subset \mathbb{C}[M] .
$$

Lastly, when $I$ is the ideal of a subvariety $X$, the initial scheme $\operatorname{in}_{w} X \subset \mathbb{T}_{N}$ is defined by the initial ideal $\operatorname{in}_{w} I$.

The sphere $\mathbb{S}_{N}:=\left(\mathbb{R}_{N} \backslash\{0\}\right) / \mathbb{R}^{+}$is the set of directions in $\mathbb{R}_{N}$. Let $\pi: \mathbb{R}_{N} \backslash\{0\} \rightarrow$ $\mathbb{S}_{N}$ be the projection. The logarithmic limit set $\mathscr{L}^{\infty}(X)$ of a subvariety $X$ of $\mathbb{T}_{N}$ is the set of accumulation points in $\mathbb{S}_{N}$ of sequences $\left\{\pi\left(\log \left(x_{n}\right)\right)\right\}$, where $\left\{x_{n}\right\} \subset X$ is an unbounded set. A sequence $\left\{x_{n}\right\} \subset \mathbb{T}_{N}$ is unbounded if its sequence of logarithms $\left\{\log \left(x_{n}\right)\right\}$ is unbounded.

A rational polyhedral cone $\sigma \subset \mathbb{R}_{N}$ is the set of points $w \in \mathbb{R}_{N}$ that satisfy finitely many inequalities and equations of the form

$$
\langle\boldsymbol{m}, w\rangle \geq 0 \quad \text { and } \quad\left\langle\boldsymbol{m}^{\prime}, w\right\rangle=0,
$$

where $\boldsymbol{m}, \boldsymbol{m}^{\prime} \in M$. The dimension of $\sigma$ is the dimension of its linear span, and faces of $\sigma$ are proper subsets of $\sigma$ obtained by replacing some inequalities by equations. The relative interior of $\sigma$ consists of its points not lying in any face. Also, $\sigma$ is determined by $\sigma \cap N$, which is a finitely generated subsemigroup of $N$.

A rational polyhedral fan $\Sigma$ is a collection of rational polyhedral cones in $\mathbb{R}_{N}$ in which every two cones of $\Sigma$ meet along a common face.

Theorem 3. The cone in $\mathbb{R}_{N}$ over the negative $-\mathscr{L}^{\infty}(X)$ of the logarithmic limit set of $X$ is the set of $w \in \mathbb{R}_{N}$ such that $\operatorname{in}_{w} X \neq \varnothing$. Equivalently, it is the set of $w \in \mathbb{R}_{N}$ such that for every $f \in \mathbb{C}[M]$ lying in the ideal $I$ of $X, \operatorname{in}_{w} f$ is not a monomial. This cone over $-\mathscr{L}^{\infty}(X)$ admits the structure of a rational polyhedral 
fan $\Sigma$ with the property that if $u$ and $w$ lie in the relative interior of a cone $\sigma$ of $\Sigma$, then $\operatorname{in}_{u} I=\operatorname{in}_{w} I$.

It is important to take $-\mathscr{L}^{\infty}(X)$. This is correct as we use the tropical convention of minimum, which is forced by our use of toric varieties to prove Theorem 1 in Section 4.2.

We write $\operatorname{in}_{\sigma} I$ for the initial ideal defined by points in the relative interior of a cone $\sigma$ of $\Sigma$. The fan structure $\Sigma$ is not canonical for it depends upon an identification $M \stackrel{\sim}{\rightarrow} \mathbb{Z}^{n}$. Moreover, it may be the case that $\sigma \neq \tau$ but $\operatorname{in}_{\sigma} I=\operatorname{in}_{\tau} I$.

Bergman [1971] defined the logarithmic limit set of a subvariety of the torus $\mathbb{T}_{N}$, and Bieri and Groves [1984] showed it was a finite union of convex polyhedral cones. The connection to initial ideals was made more explicit through work of Kapranov [2006], and the form above is adapted from Speyer and Sturmfels [2004]. The logarithmic limit set of $X$ is now called the tropical algebraic variety of $X$, and this latter work led to the field of tropical geometry.

\section{Lines in space}

We consider coamoebae of lines in three-dimensional space. We will work in the torus $\mathbb{T} \mathbb{P}^{3}$ of $\mathbb{P}^{3}$, which is the quotient of $\mathbb{T}^{4}$ by the diagonal torus $\Delta_{\mathbb{T}}$ and similarly in $\mathbb{U}^{3}$, the quotient of $\mathbb{U}^{4}$ by the diagonal $\Delta_{\mathbb{U}}:=\{(\theta, \theta, \theta, \theta) \mid \theta \in \mathbb{U}\}$. By coordinate lines and planes in $U \mathbb{P}^{3}$, we mean the images in $U \mathbb{P}^{3}$ of lines and planes in $\mathbb{U}^{4}$ parallel to some coordinate plane.

Let $\ell$ be a line in $\mathbb{P}^{3}$ not lying in any coordinate plane, so $\ell$ has a parametrization

$$
\phi: \mathbb{P}^{1} \ni[s: t] \mapsto\left[\ell_{0}(s, t): \ell_{1}(s, t): \ell_{2}(s, t): \ell_{3}(s, t)\right],
$$

where $\ell_{0}, \ell_{1}, \ell_{2}$, and $\ell_{3}$ are nonzero linear forms that do not all vanish at the same point. For $i=0, \ldots, 3$, let $\zeta_{i} \in \mathbb{P}^{1}$ be the zero of $\ell_{i}$. The configuration of these zeroes determine the coamoeba of $\ell \cap \mathbb{T} \mathbb{P}^{3}$, which we will simply write as $\operatorname{co} A(\ell)$.

Suppose that two zeroes coincide; say $\zeta_{3}=\zeta_{2}$. Then $\ell_{3}=a \ell_{2}$ for some $a \in \mathbb{C}^{*}$, and so $\ell$ lies in the translated subtorus $z_{3}=a z_{2}$, and its coamoeba $\operatorname{co} A(\ell)$ lies in the coordinate subspace of $\mathbb{U}_{3}$ defined by $\theta_{3}=\arg (a)+\theta_{2}$. In fact, $\operatorname{co} A(\ell)$ is pulled back from the coamoeba of the projection of $\ell$ to the $\theta_{3}=0$ plane. It follows that if there are only two distinct roots among $\zeta_{0}, \ldots, \zeta_{3}$, then $\operatorname{co} A(\ell)$ is a coordinate line of $\mathbb{U}_{3}$. If three of the roots are distinct, then (up to a translation) the projection of the coamoeba $\operatorname{co} \mathscr{A}(\ell)$ to the $\theta_{3}=0$ plane looks like (1) so that $\operatorname{co} \mathscr{A}(\ell)$ consists of two triangles lying in a coordinate plane.

For each $i=0, \ldots, 3$, define a function depending upon a point $[s: t] \in \mathbb{P}^{1}$ and $\theta \in \mathbb{U}$ by

$$
\varphi_{i}(s, t ; \theta):= \begin{cases}\theta & \text { if } \ell_{i}(s, t)=0 \\ \arg \left(\ell_{i}(s, t)\right) & \text { otherwise. }\end{cases}
$$


For each $i=0, \ldots, 3$, let $h_{i}$ be the image in $\mathbb{U P}^{3}$ of $\mathbb{U}$ under the map

$$
\theta \mapsto\left[\varphi_{0}\left(\zeta_{i}, \theta\right), \varphi_{1}\left(\zeta_{i}, \theta\right), \varphi_{2}\left(\zeta_{i}, \theta\right), \varphi_{3}\left(\zeta_{i}, \theta\right)\right]
$$

Lemma 4. For each $i=0, \ldots, 3, h_{i}$ is a coordinate line in $\mathbb{U P}^{3}$ that consists of accumulation points of $\operatorname{coA}(\ell)$.

This follows from Theorem 1. For the main idea, note that $\arg \circ \phi\left(\zeta_{i}+\epsilon e^{\theta \sqrt{-1}}\right)$ for $\theta \in \mathbb{U}$ is a curve in $\mathbb{U} \mathbb{P}^{3}$ whose Hausdorff distance to the line $h_{i}$ approaches 0 as $\epsilon \rightarrow 0$. The phase limit set of $\ell$ is the union of these four lines.

Lemma 5. Suppose that the zeroes $\zeta_{0}, \zeta_{1}$, and $\zeta_{2}$ are distinct. Then

$$
\mathbb{P}^{1} \backslash\left\{\zeta_{0}, \zeta_{1}, \zeta_{2}\right\} \ni x \mapsto \arg \left(\ell_{0}(x), \ell_{1}(x), \ell_{2}(x)\right) \in \mathbb{U}^{3} / \Delta_{\mathbb{U}}=\mathbb{U} \mathbb{P}^{2}
$$

is constant along each arc of the circle in $\mathbb{P}^{1}$ through $\zeta_{0}, \zeta_{1}$, and $\zeta_{2}$.

Proof. After changing coordinates in $\mathbb{P}^{1}$ and translating in $\mathbb{U P}^{2}$ (rotating coordinates), we may assume that these roots are $\infty, 0$, and -1 , and so the circle becomes the real line. Choosing affine coordinates, we may assume that $\ell_{0}=1, \ell_{1}=x$, and $\ell_{2}=x+1$ so that we are in the situation of Example 2. Then the statement of the lemma is the computation there for $x$ real in which we obtained the coordinate points $(\pi, 0),(\pi, \pi)$, and $(0, \pi)$.

Lemma 6. The phase limit lines $h_{0}, h_{1}, h_{2}$, and $h_{3}$ are disjoint if and only if the roots $\zeta_{0}, \ldots, \zeta_{3}$ do not all lie on a circle.

Proof. Suppose that two of the limit lines meet, say $h_{0}$ and $h_{1}$. Without loss of generality, we suppose that we have chosen coordinates on $\mathbb{U}^{4}$ and $\mathbb{P}^{1}$ so that $\zeta_{i} \in \mathbb{C}$ and $\ell_{i}(x)=x-\zeta_{i}$ for $i=0, \ldots, 3$. Then there are points $\alpha, \beta, \theta \in \mathbb{U}$ such that

$$
\begin{aligned}
\left(\varphi_{0}\left(\zeta_{0}, \alpha\right), \varphi_{1}\left(\zeta_{0}, \alpha\right), \varphi_{2}\left(\zeta_{0}, \alpha\right), \varphi_{3}\left(\zeta_{0}, \alpha\right)\right) & \\
& =\left(\varphi_{0}\left(\zeta_{1}, \beta\right), \varphi_{1}\left(\zeta_{1}, \beta\right), \varphi_{2}\left(\zeta_{1}, \beta\right), \varphi_{3}\left(\zeta_{1}, \beta\right)\right)+(\theta, \theta, \theta, \theta) .
\end{aligned}
$$

Comparing the last two components, we obtain

$$
\arg \left(\zeta_{0}-\zeta_{2}\right)=\arg \left(\zeta_{1}-\zeta_{2}\right)+\theta \quad \text { and } \quad \arg \left(\zeta_{0}-\zeta_{3}\right)=\arg \left(\zeta_{1}-\zeta_{3}\right)+\theta
$$

and so the zeroes $\zeta_{0}, \ldots, \zeta_{3}$ have the configuration below:

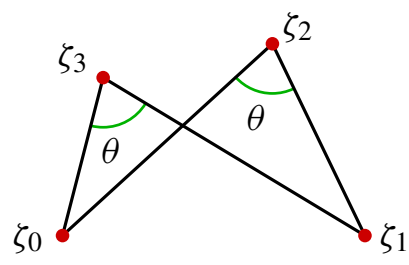


But then $\zeta_{0}, \ldots, \zeta_{3}$ are cocircular. Conversely, if $\zeta_{0}, \ldots, \zeta_{3}$ lie on a circle $C$, then by Lemma 5 , the lines $h_{i}$ and $h_{j}$ meet only if $\zeta_{i}$ and $\zeta_{j}$ are the endpoints of an arc of $C \backslash\left\{\zeta_{0}, \ldots, \zeta_{3}\right\}$.

Lemma 7. If the roots $\zeta_{0}, \ldots, \zeta_{3}$ do not all lie on a circle, then the map

$$
\arg \circ \phi: \mathbb{P}^{1} \backslash\left\{\zeta_{0}, \zeta_{1}, \zeta_{2}, \zeta_{3}\right\} \rightarrow \mathbb{U} \mathbb{P}^{3}
$$

is an immersion.

Proof. Let $x \in \mathbb{P}^{1} \backslash\left\{\zeta_{0}, \zeta_{1}, \zeta_{2}, \zeta_{3}\right\}$, which we consider to be a real two-dimensional manifold. After possibly reordering the roots, the circle $C_{1}$ containing $x, \zeta_{0}$, and $\zeta_{1}$ meets the circle $C_{2}$ containing $x, \zeta_{2}$, and $\zeta_{3}$ transversally at $x$. Under the derivative of the map $\arg \circ \phi$, tangent vectors at $x$ to $C_{1}$ and $C_{2}$ are taken to nonzero vectors $\left(0,0, u_{1}, v_{1}\right)$ and $\left(u_{2}, v_{2}, 0,0\right)$ in the tangent space to $\mathbb{U}^{4}$. Furthermore, as the four roots do not all lie on a circle, we cannot have both $u_{1}=v_{1}$ and $u_{2}=v_{2}$, and so this derivative has full rank two at $x$ as a map from $\mathbb{P}^{1} \backslash\left\{\zeta_{0}, \zeta_{1}, \zeta_{2}, \zeta_{3}\right\} \rightarrow \mathbb{U} \mathbb{P}^{3}$, which proves the lemma.

By these lemmas, there is a fundamental difference between the coamoebae of lines when the roots of the linear forms $\ell_{i}$ are cocircular and when they are not. We examine each case in detail. First, choose coordinates so that $\zeta_{0}=\infty$. After dehomogenizing and separately rescaling each affine coordinate (e.g., identifying $\mathbb{U} \mathbb{P}^{3}$ with $\mathbb{U}^{3}$ and applying phase shifts to each coordinate $\theta_{1}, \theta_{2}, \theta_{3}$ of $\left.\mathbb{U}^{3}\right)$, we may assume that the map (3) parametrizing $\ell$ is

$$
\phi: \mathbb{C} \ni x \mapsto\left(x-\zeta_{1}, x-\zeta_{2}, x-\zeta_{3}\right) \in \mathbb{C}^{3} .
$$

Suppose first that the four roots are cocircular. As $z_{0}=\infty$, the other three lie on a real line in $\mathbb{C}$, which we may assume is $\mathbb{R}$. That is, if the four roots are cocircular, then up to coordinate change, we may assume that the line $\ell$ is real and the affine parametrization (4) is also real. For this reason, we will call such lines $\ell$ real lines. We first study the boundary of $\operatorname{co} A(\ell)$. Suppose that $x$ lies on a contour $C$ in the upper half plane as in Figure 1 that contains semicircles of radius $\epsilon$ centered at each root and a semicircle of radius $1 / \epsilon$ centered at 0 but otherwise lies along the real

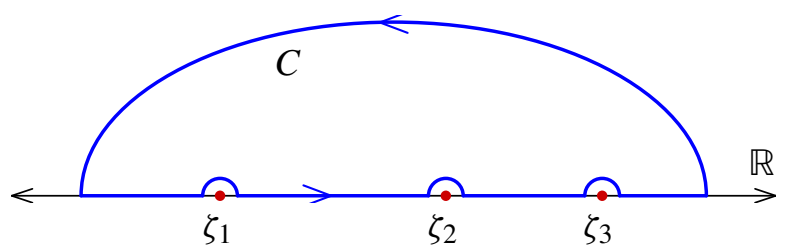

Figure 1. Contour in upper half plane. 
axis for $\epsilon$, a sufficiently small positive number. Then $\arg (\phi(w)) \in \mathbb{U}^{3}$ is constant on the four segments of $C$ lying along $\mathbb{R}$ with respective values

$$
(\pi, \pi, \pi), \quad(0, \pi, \pi), \quad(0,0, \pi), \quad \text { and } \quad(0,0,0)
$$

moving from left to right. On the semicircles around $\zeta_{1}, \zeta_{2}$, and $\zeta_{3}$, two of the coordinates are essentially constant (but not quite equal to either 0 or $\pi$ !) while the third decreases from $\pi$ to 0 . Finally, on the large semicircle, the three coordinates are nearly equal and increase from $(0,0,0)$ to $(\pi, \pi, \pi)$. The image $\arg (\phi(C))$ can be made as close as we please to the quadrilateral in $\mathbb{U}^{3}$ connecting the points of (5) in cyclic order when $\epsilon$ is sufficiently small. Thus, the image of the upper half plane under the map $\arg \circ \phi$ is a relatively open membrane in $\mathbb{U}^{3}$ that spans the quadrilateral. It lies within the convex hull of this quadrilateral, which is computed using the affine structure induced from $\mathbb{R}^{3}$ by the quotient $\mathbb{U}^{3}=\mathbb{R}^{3} /(2 \pi \mathbb{Z})^{3}$.

For this, observe that its projection in any of the four coordinate directions parallel to its edges is one of the triangles of the coamoeba of the projected line in $\mathbb{C P}^{2}$ of Example 2, and the convex hull of the quadrilateral is the intersection of the four preimages of these triangles.

Because $\ell$ is real, the image of the lower half plane is isomorphic to the image of the upper half plane under the map $\left(\theta_{1}, \theta_{2}, \theta_{3}\right) \mapsto\left(-\theta_{1},-\theta_{2},-\theta_{3}\right)$, and so the coamoeba is symmetric in the origin of $\mathbb{U}^{3}$ and consists of two quadrilateral patches that meet at their vertices. Here are two views of the coamoeba of the line where the roots are $\infty,-1 / 2,0$, and $3 / 2$ :
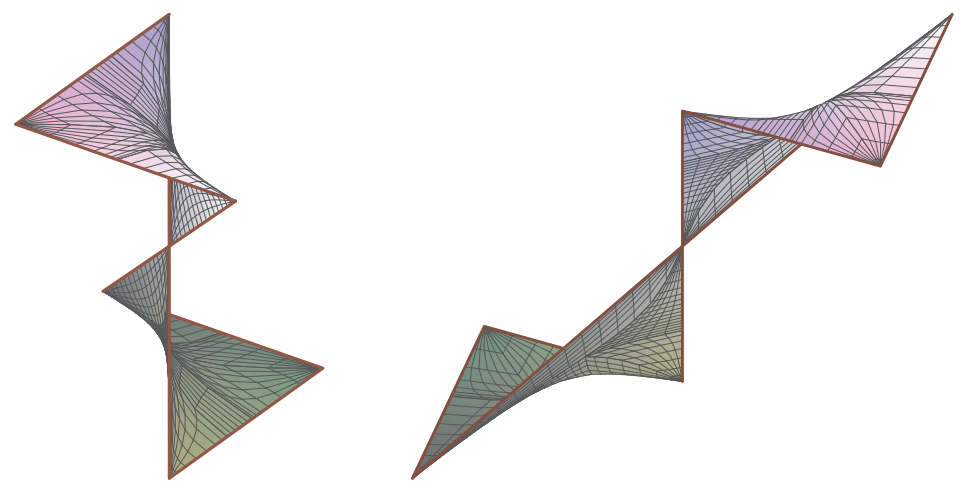

Now suppose that the roots $\zeta_{0}, \ldots, \zeta_{3}$ do not all lie on a circle. By Lemma 6, the four phase limit lines $h_{1}, \ldots, h_{3}$ are disjoint, and the map from $\ell$ to the coamoeba is an immersion. Figure 2 shows two views of the coamoeba in a fundamental domain of $U \mathbb{P}^{3}$ when the roots are $\infty, 1, \zeta$, and $\zeta^{2}$, where $\zeta$ is a primitive third root of infinity. This and other pictures of coamoebae of lines are animated on the website [Nisse and Sottile 2010]. 

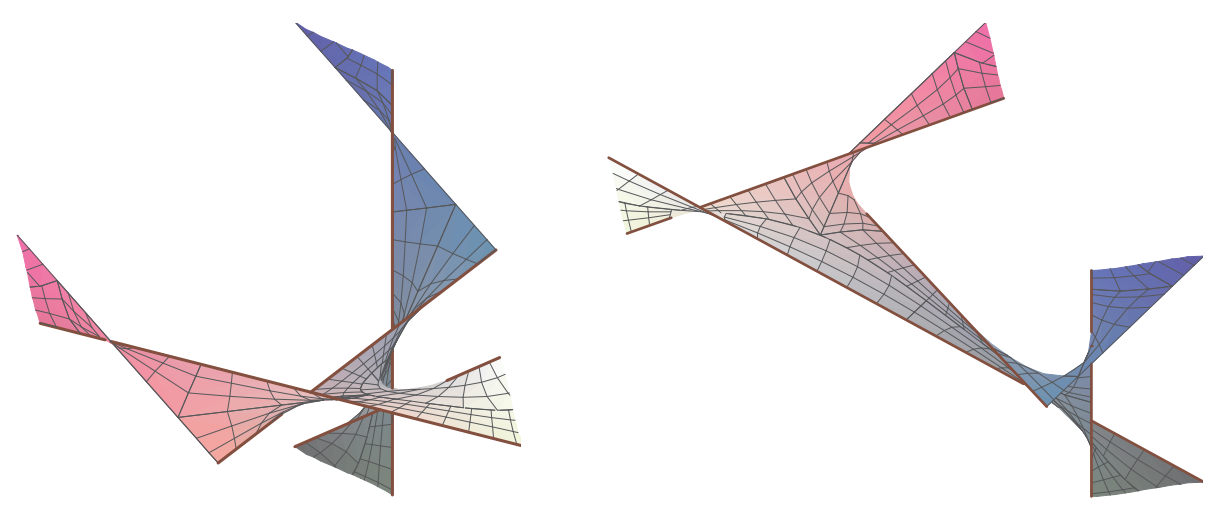

Figure 2. Two views of the coamoeba of a symmetric line.

The projection of this coamoeba along a coordinate direction (parallel to one of the phase limit lines $h_{i}$ ) gives a coamoeba of a line in $\mathbb{\mathbb { P } ^ { 2 }}$ as we saw in Example 2. The line $h_{i}$ is mapped to the interior of one triangle, and the vertices of the triangles are the images of line segments lying on the coamoeba. These three line segments come from the three arcs of the circle through the three roots other than $\zeta_{i}$, the root corresponding to $h_{i}$.

Proposition 8. The interior of the coamoeba of a general line in $\mathbb{\mathbb { P } ^ { 3 }}$ contains twelve line segments in triples parallel to each of the four coordinate directions.

The symmetric coamoeba we show in Figure 2 has six additional line segments, two each coming from the three longitudinal circles through a third root of unity and 0 and $\infty$. Two such segments are visible as pinch points in the leftmost view in Figure 2. We ask, What is the maximal number of line segments on a coamoeba of a line in $\mathbb{T} \mathbb{P}^{3}$ ?

\section{Structure of the phase limit set}

The phase limit set $\mathscr{P}^{\infty}(X)$ of a complex subvariety $X \subset \mathbb{T}_{N}$ is the set of all accumulation points of sequences $\left\{\arg \left(x_{n}\right) \mid n \in \mathbb{N}\right\} \subset \mathbb{U}_{N}$, where $\left\{x_{n} \mid n \in \mathbb{N}\right\} \subset X$ is an unbounded sequence. For $w \in N, \operatorname{in}_{w} X \subset \mathbb{T}_{N}$ is the (possibly empty) initial scheme of $X$, whose ideal is the initial ideal $\operatorname{in}_{w} I$, where $I$ is the ideal of $X$. Our main result, Theorem 1, is that the phase limit set of $X$ is the union of the coamoebae of all its initial schemes.

Remark 9. The union of Theorem 1 is finite. By Theorem 3, $\operatorname{in}_{w} X$ is nonempty only when $w$ lies in the cone over the logarithmic set $\mathscr{L}^{\infty}(X)$, which can be given the structure of a finite union of rational polyhedral cones such that any two points in the relative interior of the same cone $\sigma$ have the same initial scheme. If we write $\operatorname{in}_{\sigma} X$ for the initial scheme corresponding to a cone $\sigma$, the torus $\mathbb{T}_{\langle\sigma\rangle} \simeq\left(\mathbb{C}^{*}\right)^{\operatorname{dim} \sigma}$ 
acts on $\operatorname{in}_{\sigma} X$ by translation (e.g., see Corollary 13). (Here, $\langle\sigma\rangle \subset N$ is the span of $\sigma \cap N$, a free abelian group of rank $\operatorname{dim} \sigma$.) This implies $\operatorname{co} A\left(\operatorname{in}_{\sigma} X\right)$ is a union of orbits of $\operatorname{coA}\left(\mathbb{T}_{\langle\sigma\rangle}\right)=\mathbb{U}_{\langle\sigma\rangle}$ and thus that $\operatorname{dim}\left(\cos \left(\operatorname{in}_{\sigma} X\right)\right) \leq 2 \operatorname{dim}(X)-\operatorname{dim}(\sigma)$.

This discussion implies the following proposition:

Proposition 10. Let $X \subset \mathbb{T}_{N}$ be a subvariety, and suppose that $\mathbb{T}_{X} \subset \mathbb{T}_{N}$ is the largest subtorus acting on $X$. Then $\operatorname{dim} \operatorname{co} A(X) \leq \min \left\{\operatorname{dim} \mathbb{T}_{N}, 2 \operatorname{dim} X-\operatorname{dim} \mathbb{T}_{X}\right\}$.

We prove Theorem 1 in the next two subsections.

4.1. Coamoebae of initial schemes. We review the standard dictionary relating initial ideals to toric degenerations in the context of subvarieties of $\mathbb{T}_{N}$ [Gelfand et al. 1994, Chapter 6]. Let $X \subset \mathbb{T}_{N}$ be a subvariety with ideal $I \subset \mathbb{C}[M]$. We study $\operatorname{in}_{w} I$ and the initial schemes $\operatorname{in}_{w} X=\mathscr{V}\left(\operatorname{in}_{w} I\right) \subset \mathbb{T}_{N}$ for $w \in N$. Since $\operatorname{in}_{0} I=I$ so that $\operatorname{in}_{0} X=X$, we may assume that $w \neq 0$. As $N$ is the lattice of oneparameter subgroups of $\mathbb{T}_{N}, w$ corresponds to a one-parameter subgroup written as $\mathbb{C}^{*} \ni t \mapsto t^{w} \in \mathbb{T}_{N}$. Define $\mathscr{X} \subset \mathbb{C} \times \mathbb{T}_{N}$ by

$$
\mathscr{L}:=\left\{(t, x) \in \mathbb{C}^{*} \times \mathbb{T}_{N} \mid t^{w} \cdot x \in X\right\} .
$$

The fiber of $\mathscr{X}$ over a point $t \in \mathbb{C}^{*}$ is $t^{-w} X$. Let $\overline{\mathscr{X}}$ be the closure of $\mathscr{X}$ in $\mathbb{C} \times \mathbb{T}_{N}$, and set $X_{0}$ to be the fiber of $\overline{\mathscr{X}}$ over $0 \in \mathbb{C}$.

Proposition 11. $X_{0}=\operatorname{in}_{w} X$.

Proof. We first describe the ideal $\mathscr{I}$ of $\mathscr{X}$. For $\boldsymbol{m} \in M$, the element $\xi^{\boldsymbol{m}} \in \mathbb{C}[M]$ takes the value $t^{\langle\boldsymbol{m}, w\rangle} \in \mathbb{C}^{*}$ on the element $t^{w} \in \mathbb{T}_{N}$, and so if $x \in \mathbb{T}_{N}$, then $\xi^{\boldsymbol{m}}$ takes the value $t^{\langle\boldsymbol{m}, w\rangle} \xi^{\boldsymbol{m}}(x)=t^{\langle\boldsymbol{m}, w\rangle} \boldsymbol{m}(x)$ on $t^{w} x$. Given a polynomial $f \in \mathbb{C}[M]$ of the form

$$
f:=\sum_{\boldsymbol{m} \in \mathscr{A}} c_{\boldsymbol{m}} \xi^{\boldsymbol{m}} \text { for } c_{\boldsymbol{m}} \in \mathbb{C}^{*}
$$

define the polynomial $f(t) \in \mathbb{C}\left[t, t^{-1}\right][M]$ by

$$
f(t):=\sum_{\boldsymbol{m} \in \mathscr{A}} c_{\boldsymbol{m}} t^{\langle\boldsymbol{m}, w\rangle} \xi^{\boldsymbol{m}}
$$

Then $f(t)(x)=f\left(t^{w} x\right)$, so $\mathscr{I}$ is generated by the polynomials $f(t)$ of (7) for $f \in I$. A general element of $\mathscr{I}$ is a linear combination of translates $t^{a} f(t)$ of such polynomials for $a \in \mathbb{Z}$.

If we set $w(f)$ to be the minimal exponent of $t$ occurring in $f(t)$, then

$$
\operatorname{in}_{w} f=\sum_{\langle\boldsymbol{m}, w\rangle=w(f)} c_{\boldsymbol{m}} \xi^{\boldsymbol{m}}
$$

and

$$
t^{-w(f)} f(t)=\operatorname{in}_{w} f+\sum_{\langle\boldsymbol{m}, w\rangle>w(f)} t^{\langle\boldsymbol{m}, w\rangle-w(f)} c_{\boldsymbol{m}} \xi^{\boldsymbol{m}}
$$




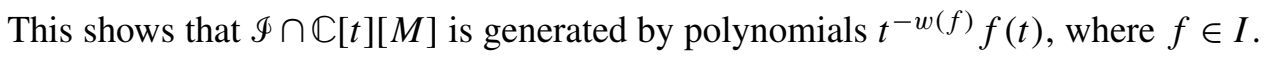
Since $\operatorname{in}_{w} f \in \mathbb{C}[M]$ and the remaining terms are divisible by $t$, we see that the ideal of $X_{0}$ is generated by $\left\{\operatorname{in}_{w} f \mid f \in \mathscr{Y}\right\}$, which completes the proof.

We use Proposition 11 to prove one inclusion of Theorem 1, namely that

$$
\mathscr{P}^{\infty}(X) \supset \bigcup_{w \in N \backslash\{0\}} \operatorname{coA}\left(\mathrm{in}_{w} X\right) .
$$

Fix $0 \neq w \in N$, and let $\mathscr{X}, \overline{\mathscr{X}}$, and $X_{0}=\operatorname{in}_{w} X$ be as in Proposition 11, and let $x_{0} \in X_{0}$. We show that $\arg \left(x_{0}\right) \in \mathscr{P}^{\infty}(X)$. Since $\left(0, x_{0}\right) \in \overline{\mathscr{X}}$, there is an irreducible curve $C \subset \mathscr{X}$ with $\left(0, x_{0}\right) \in \bar{C}$. The projection of $C \subset \mathbb{C}^{*} \times \mathbb{T}_{N}$ to $\mathbb{C}^{*}$ is dominant, so there exists a sequence $\left\{\left(t_{n}, x_{n}\right) \mid n \in \mathbb{N}\right\} \subset C$ that converges to $\left(0, x_{0}\right)$ with each $t_{n}$ real and positive. Then $\arg \left(x_{0}\right)$ is the limit of the sequence $\left\{\arg \left(x_{n}\right)\right\}$.

For each $n \in \mathbb{N}$, set $y_{n}:=t_{n}^{w} \cdot x_{n} \in X$. Since $t_{n}$ is positive and real, every component of $t_{n}^{w}$ is positive and real, and so $\arg \left(y_{n}\right)=\arg \left(x_{n}\right)$. Thus, $\arg \left(x_{0}\right)$ is the limit of the sequence $\left\{\arg \left(y_{n}\right)\right\}$. Since $x_{n}$ converges to $x_{0}$ and $t_{n}$ converges to 0 , the sequence $\left\{y_{n}\right\} \subset X$ is unbounded, which implies that $\arg \left(x_{0}\right)$ lies in the phase limit set of $X$. This proves (8).

4.2. Coamoebae and tropical compactifications. We finish the proof of Theorem 1 by establishing the other inclusion,

$$
\mathscr{P}^{\infty}(X) \subset \bigcup_{w \in N \backslash\{0\}} \operatorname{co} \mathscr{A}\left(\operatorname{in}_{w} X\right) .
$$

Suppose that $\left\{x_{n} \mid n \in \mathbb{N}\right\} \subset X$ is an unbounded sequence. To study the accumulation points of the sequence $\left\{\arg \left(x_{n}\right) \mid n \in \mathbb{N}\right\}$, we use a compactification of $X$ that is adapted to its inclusion in $\mathbb{T}_{N}$. Suitable compactifications are the tropical compactifications of Tevelev [2007] for in these the boundary of $X$ is composed of initial schemes $\operatorname{in}_{w} X$ of $X$ in a manner we describe below.

By Theorem 3, the cone over the logarithmic limit set $\mathscr{L}^{\infty}(X)$ of $X$ is the support of a rational polyhedral fan $\Sigma$ whose cones $\sigma$ have the property that all initial ideals $\operatorname{in}_{w} I$ coincide for $w$ in the relative interior of $\sigma$.

Recall the construction of the toric variety $Y_{\Sigma}$ associated with a fan $\Sigma$ [Fulton 1993; Gelfand et al. 1994, Chapter 6]. For $\sigma \in \Sigma$, set

$$
\begin{aligned}
\sigma^{\vee} & :=\{\boldsymbol{m} \in M \mid\langle\boldsymbol{m}, w\rangle \geq 0 \text { for all } w \in \sigma\}, \\
\sigma^{\perp} & :=\{\boldsymbol{m} \in M \mid\langle\boldsymbol{m}, w\rangle=0 \text { for all } w \in \sigma\} .
\end{aligned}
$$

Set $V_{\sigma}:=\operatorname{spec} \mathbb{C}\left[\sigma^{\vee}\right]$ and $\sigma_{\sigma}:=\operatorname{spec} \mathbb{C}\left[\sigma^{\perp}\right]$, which is naturally isomorphic to $\mathbb{\mathbb { T }}_{N} / \mathbb{\mathbb { T }}_{\langle\sigma\rangle}$, where $\langle\sigma\rangle \subset N$ is the subgroup generated by $\sigma \cap N$. The map $\boldsymbol{m} \mapsto \boldsymbol{m} \otimes \boldsymbol{m}$ determines a comodule map $\mathbb{C}\left[\sigma^{\vee}\right] \rightarrow \mathbb{C}\left[\sigma^{\vee}\right] \otimes \mathbb{C}[M]$, which induces the action of 
the torus $\mathbb{\mathbb { T }}_{N}$ on $V_{\sigma}$. Its orbits correspond to faces of the cone $\sigma$, and the smallest orbit $\mathrm{O}_{\sigma}$ corresponds to $\sigma$ itself. The inclusion $\sigma^{\perp} \subset \sigma^{\vee}$ is split by the semigroup map

$$
\sigma^{\vee} \ni \boldsymbol{m} \mapsto \begin{cases}\boldsymbol{m} & \text { if } \boldsymbol{m} \in \sigma^{\perp}, \\ 0 & \text { if } \boldsymbol{m} \notin \sigma^{\perp},\end{cases}
$$

which induces a map $\mathbb{C}[M] \rightarrow \mathbb{C}\left[\sigma^{\perp}\right]$, and thus, we have the $\mathbb{\mathbb { T }}_{N}$-equivariant split inclusion

$$
\mathrm{O}_{\sigma} \longleftrightarrow V_{\sigma} \stackrel{\pi_{\sigma}}{\longrightarrow} \mathrm{O}_{\sigma} .
$$

On orbits $\hat{O}_{\tau}$ in $V_{\sigma}$, the map $\pi_{\sigma}$ is simply the quotient by $\mathbb{T}_{\langle\sigma\rangle}$.

If $\sigma, \tau \in \Sigma$ with $\sigma \subset \tau$, then $\sigma^{\vee} \supset \tau^{\vee}$, so $\mathbb{C}\left[\sigma^{\vee}\right] \supset \mathbb{C}\left[\tau^{\vee}\right]$, and so $V_{\sigma} \subset V_{\tau}$. Since the quotient fields of $\mathbb{C}\left[\sigma^{\vee}\right]$ and $\mathbb{C}[M]$ coincide, these are inclusions of open sets, and these varieties $V_{\sigma}$ for $\sigma \in \Sigma$ glue together along these natural inclusions to give the toric variety $Y_{\Sigma}$. The torus $\mathbb{T}_{N}$ acts on $Y_{\Sigma}$ with an orbit $\sigma_{\sigma}$ for each cone $\sigma$ of $\Sigma$.

Since $V_{0}=\mathbb{\mathbb { T }}_{N}, Y_{\Sigma}$ contains $\mathbb{\mathbb { T }}_{N}$ as a dense subset, and thus $X$ is a (nonclosed) subvariety. Let $\bar{X}$ be the closure of $X$ in $Y_{\Sigma}$. As the fan $\Sigma$ is supported on the cone over $\mathscr{L}^{\infty}(X), \bar{X}$ will be a tropical compactification of $X$, and $\bar{X}$ is complete [Tevelev 2007, Proposition 2.3]. To understand the points of $\bar{X} \backslash X$, we study the intersection $\bar{X} \cap V_{\sigma}$, which is defined by $I \cap \mathbb{C}\left[\sigma^{\vee}\right]$, as well as the intersection $\bar{X} \cap \mathscr{O}_{\sigma}$, which is defined in $\mathbb{C}\left[\sigma^{\perp}\right]$ by the image $I(\sigma)$ of $I \cap \mathbb{C}\left[\sigma^{\vee}\right]$ under the map $\mathbb{C}\left[\sigma^{\vee}\right] \rightarrow \mathbb{C}\left[\sigma^{\perp}\right]$ induced by $(10)$.

Lemma 12. The initial ideal $\operatorname{in}_{\sigma} I \subset \mathbb{C}[M]$ of $I$ is generated by $I(\sigma)$ under the inclusion $\mathbb{C}\left[\sigma^{\perp}\right] \hookrightarrow \mathbb{C}[M]$.

Proof. Let $f \in I$. Since $\sigma$ is a cone in $\Sigma$, we have that $\operatorname{in}_{\sigma} f=\operatorname{in}_{w} f$ for all $w$ in the relative interior of $\sigma$. Thus, for $w \in \sigma$, the function $\boldsymbol{m} \mapsto\langle\boldsymbol{m}, w\rangle$ on exponents of monomials of $f$ is minimized on (a superset of) the support of $\operatorname{in}_{\sigma} f$, and if $w$ lies in the relative interior of $\sigma$, then the minimizing set is the support of $\operatorname{in}_{\sigma} f$. Multiplying $f$ if necessary by $\xi^{-\boldsymbol{m}}$, where $\boldsymbol{m}$ is some monomial of $\operatorname{in}_{\sigma} f$, we may assume that for every $w \in \sigma$, the linear function $\boldsymbol{m} \mapsto\langle\boldsymbol{m}, w\rangle$ is nonnegative on the support of $f$ so that $f \in \mathbb{C}\left[\sigma^{\vee}\right]$, and the function is zero on the support of $\operatorname{in}_{\sigma} f$. Furthermore, if $w$ lies in the relative interior of $\sigma$, then it vanishes exactly on the support of $\operatorname{in}_{\sigma} f$. Thus, $\operatorname{in}_{\sigma} f \in \mathbb{C}\left[\sigma^{\perp}\right]$, which completes the proof.

Since $\mathscr{O}_{\sigma}=\mathbb{T}_{N} / \mathbb{T}_{\langle\sigma\rangle}$, Lemma 12 has the following geometric counterpart:

Corollary 13. By translation with $\operatorname{in}_{\sigma} X / \mathbb{T}_{\langle\sigma\rangle}=\bar{X} \cap \mathcal{O}_{\sigma}, \mathbb{T}_{\langle\sigma\rangle}$ acts (freely) on $\operatorname{in}_{\sigma} X$.

Proof of Theorem 1. Let $\theta \in \mathscr{P}^{\infty}(X)$ be a point in the phase limit set of $X$. Then there exists an unbounded sequence $\left\{x_{n} \mid n \in \mathbb{N}\right\} \subset X$ with

$$
\lim _{n \rightarrow \infty} \arg \left(x_{n}\right)=\theta
$$


Since $\bar{X}$ is compact, the sequence $\left\{x_{n} \mid n \in \mathbb{N}\right\}$ has an accumulation point $x$ in $\bar{X}$. As the sequence is unbounded, $x \notin \mathbb{O}_{0}$, and so $x \in \bar{X} \backslash X$. Thus, $x$ is a point of $\bar{X} \cap \mathcal{O}_{\sigma}$ for some cone $\sigma \neq 0$ of $\Sigma$. Replacing $\left\{x_{n}\right\}$ by a subsequence, we may assume that $\lim _{n} x_{n}=x$.

Because the map $\pi_{\sigma}$ of (10) is continuous and is the identity on $\mathbb{O}_{\sigma}$, we have that $\left\{\pi_{\sigma}\left(x_{n}\right)\right\}$ converges to $\pi_{\sigma}(x)=x$, and thus,

$$
\pi_{\sigma}(\theta)=\pi_{\sigma}\left(\lim _{n \rightarrow \infty} \arg \left(x_{n}\right)\right)=\arg \left(\lim _{n \rightarrow \infty} \pi_{\sigma}\left(x_{n}\right)\right)=\arg (x) \in \operatorname{co} A\left(\bar{X} \cap \bigcirc_{\sigma}\right) .
$$

Corollary 13 implies that $\operatorname{coA}\left(\bar{X} \cap \mathbb{O}_{\sigma}\right)=\operatorname{coA}\left(\operatorname{in}_{\sigma} X\right) / \mathbb{U}_{\sigma}$ as $\mathbb{U}_{\langle\sigma\rangle}=\arg \left(\mathbb{T}_{\langle\sigma\rangle}\right)$. Recall that on $\mathrm{O}_{0}, \pi_{\sigma}$ is the quotient by $\mathbb{T}_{\langle\sigma\rangle}$. Thus, we conclude from (11) that $\theta \in \operatorname{co} A\left(\mathrm{in}_{\sigma} X\right)$, which completes the proof of Theorem 1 as $\operatorname{in}_{\sigma} X=\operatorname{in}_{w} X$ for any $w$ in the relative interior of $\sigma$.

Example 14. In [Nisse 2009], the closure of a hypersurface coamoeba $\operatorname{co} \mathscr{A}(\mathscr{V}(f))$ for $f \in \mathbb{C}[M]$ was shown to contain a finite collection of codual hyperplanes. These are translates of codimension- 1 subtori $\mathbb{U}_{\sigma}$ for $\sigma$ a cone in the normal fan of the Newton polytope of $f$ corresponding to an edge. By Theorem 1, these translated tori are that part of the phase limit set of $X$ corresponding to the cones $\sigma$ dual to the edges, specifically $\operatorname{co} \mathscr{A}\left(\operatorname{in}_{\sigma} X\right)$. Since $\sigma$ has dimension $n-1$, the torus $\mathbb{T}_{\sigma}$ acts with finitely many orbits on $\operatorname{in}_{\sigma} X$, which is therefore a union of finitely many translates of $\mathbb{T}_{\sigma}$. Thus, $\operatorname{coA}\left(\operatorname{in}_{\sigma} X\right)$ is a union of finitely many translates of $\mathbb{U}_{\sigma}$.

The logarithmic limit set $\mathscr{L}^{\infty}(C)$ of a curve $C \subset \mathbb{T}_{N}$ is a finite collection of points in $\mathbb{S}_{N}$. Each point gives a ray in the cone over $\mathscr{L}^{\infty}(C)$, and the components of $\mathscr{P}^{\infty}(C)$ corresponding to a ray $\sigma$ are finitely many translations of the dimension-1 subtorus $\mathbb{U}_{\sigma}$ of $\mathbb{U}_{N}$, which we referred to as lines in Section 3. These were the lines lying in the boundaries of the coamoebae $\operatorname{co} A(\ell)$ of the lines $\ell$ in $\mathbb{T}^{2}$ and $\mathbb{T}^{3}$.

\section{References}

[Basu et al. 2006] S. Basu, R. Pollack, and M.-F. Roy, Algorithms in real algebraic geometry, 2nd ed., Algorithms and Computation in Mathematics 10, Springer, Berlin, 2006. MR 2007b:14125 Zbl 1102.14041

[Below et al. 2003] A. Below, V. Krummeck, and J. Richter-Gebert, "Complex matroids phirotopes and their realizations in rank 2", pp. 203-233 in Discrete and computational geometry, edited by B. Aronov et al., Algorithms Combin. 25, Springer, Berlin, 2003. MR 2005d:05041 Zbl 1077.52521

[Bergman 1971] G. M. Bergman, "The logarithmic limit-set of an algebraic variety", Trans. Amer. Math. Soc. 157 (1971), 459-469. MR 43 \#6209 Zbl 0197.17102

[Bieri and Groves 1984] R. Bieri and J. R. J. Groves, "The geometry of the set of characters induced by valuations", J. Reine Angew. Math. 347 (1984), 168-195. MR 86c:14001 Zbl 0526.13003

[Einsiedler et al. 2006] M. Einsiedler, M. Kapranov, and D. Lind, "Non-Archimedean amoebas and tropical varieties", J. Reine Angew. Math. 601 (2006), 139-157. MR 2007k:14038 Zbl 1115.14051

[Fulton 1993] W. Fulton, Introduction to toric varieties, Annals of Mathematics Studies 131, Princeton University Press, 1993. MR 94g:14028 Zbl 0813.14039 
[Gelfand et al. 1994] I. M. Gelfand, M. M. Kapranov, and A. V. Zelevinsky, Discriminants, resultants, and multidimensional determinants, Birkhäuser, Boston, MA, 1994. MR 95e:14045 Zbl 0827.14036

[Johansson 2013] P. Johansson, "The argument cycle and the coamoeba", Complex Variables and Elliptic Equations 58:3 (2013), 373-384.

[Kenyon et al. 2006] R. Kenyon, A. Okounkov, and S. Sheffield, "Dimers and amoebae", Ann. of Math. (2) 163:3 (2006), 1019-1056. MR 2007f:60014 Zbl 1154.82007

[Mikhalkin 2000] G. Mikhalkin, "Real algebraic curves, the moment map and amoebas", Ann. of Math. (2) 151:1 (2000), 309-326. MR 2001c:14083 Zbl 1073.14555

[Nilsson and Passare 2010] L. Nilsson and M. Passare, "Discriminant coamoebas in dimension two", J. Commut. Algebra 2:4 (2010), 447-471. MR 2011k:14033 Zbl 1237.14062

[Nisse 2009] M. Nisse, "Geometric and combinatorial structure of hypersurface coamoebas", preprint, 2009. arXiv 0906.2729

[Nisse and Sottile 2010] M. Nisse and F. Sottile, "Coamoebae of lines in 3-space", website, 2010, available at http://www.math.tamu.edu/ sottile/research/stories/coAmoeba/.

[Passare and Rullgård 2004] M. Passare and H. Rullgård, "Amoebas, Monge-Ampère measures, and triangulations of the Newton polytope", Duke Math. J. 121:3 (2004), 481-507. MR 2005a:32005 Zbl 1043.32001

[Purbhoo 2008] K. Purbhoo, "A Nullstellensatz for amoebas”, Duke Math. J. 141:3 (2008), 407-445. MR 2009b:14114 Zbl 1233.14036

[Speyer and Sturmfels 2004] D. Speyer and B. Sturmfels, "The tropical Grassmannian", Adv. Geom. 4:3 (2004), 389-411. MR 2005d:14089 Zbl 1065.14071

[Tevelev 2007] J. Tevelev, "Compactifications of subvarieties of tori", Amer. J. Math. 129:4 (2007), 1087-1104. MR 2008f:14068 Zbl 1154.14039

[Theobald 2002] T. Theobald, "Computing amoebas", Experiment. Math. 11:4 (2002), 513-526. MR 2004b:14100 Zbl 1100.14048

Communicated by Bernd Sturmfels

Received 2011-06-07 Revised 2012-02-14 Accepted 2012-03-16

nisse@math.tamu.edu

sottile@math.tamu.edu
Department of Mathematics, Texas A\&M University, College Station, TX 77843, United States http://www.math.tamu.edu/ nisse/

Department of Mathematics, Texas A\&M University, College Station, TX 77843, United States http://www. math.tamu.edu/ sottile/ 


\section{Algebra \& Number Theory}

msp.org/ant

\section{EDITORS}

MANAGING EDITOR

Bjorn Poonen

Massachusetts Institute of Technology

Cambridge, USA

\author{
EDITORIAL BOARD CHAIR \\ David Eisenbud \\ University of California \\ Berkeley, USA
}

\section{BOARD OF EDITORS}

Georgia Benkart

Dave Benson

Richard E. Borcherds

John H. Coates

J-L. Colliot-Thélène

Brian D. Conrad

Hélène Esnault

Hubert Flenner

Edward Frenkel

Andrew Granville

Joseph Gubeladze

Ehud Hrushovski

Craig Huneke

Mikhail Kapranov

Yujiro Kawamata

János Kollár

Yuri Manin

Barry Mazur

Philippe Michel
University of Wisconsin, Madison, USA

University of Aberdeen, Scotland

University of California, Berkeley, USA

University of Cambridge, UK

CNRS, Université Paris-Sud, France

University of Michigan, USA

Freie Universität Berlin, Germany

Ruhr-Universität, Germany

University of California, Berkeley, USA

Université de Montréal, Canada

San Francisco State University, USA

Hebrew University, Israel

University of Virginia, USA

Yale University, USA

University of Tokyo, Japan

Princeton University, USA

Northwestern University, USA

Harvard University, USA

École Polytechnique Fédérale de Lausanne
Susan Montgomery

Shigefumi Mori

Raman Parimala

Jonathan Pila

Victor Reiner

Karl Rubin

Peter Sarnak

Joseph H. Silverman

Michael Singer

Vasudevan Srinivas

J. Toby Stafford

Bernd Sturmfels

Richard Taylor

Ravi Vakil

Michel van den Bergh

Marie-France Vignéras

Kei-Ichi Watanabe

Efim Zelmanov
University of Southern California, USA

RIMS, Kyoto University, Japan

Emory University, USA

University of Oxford, UK

University of Minnesota, USA

University of California, Irvine, USA

Princeton University, USA

Brown University, USA

North Carolina State University, USA

Tata Inst. of Fund. Research, India

University of Michigan, USA

University of California, Berkeley, USA

Harvard University, USA

Stanford University, USA

Hasselt University, Belgium

Université Paris VII, France

Nihon University, Japan

University of California, San Diego, USA

\section{PRODUCTION}

production@msp.org

Silvio Levy, Scientific Editor

See inside back cover or msp.org/ant for submission instructions.

The subscription price for 2013 is US \$200/year for the electronic version, and \$350/year ( $\$ 40$, if shipping outside the US) for print and electronic. Subscriptions, requests for back issues and changes of subscribers address should be sent to MSP.

Algebra \& Number Theory (ISSN 1944-7833 electronic, 1937-0652 printed) at Mathematical Sciences Publishers, 798 Evans Hall \#3840, c/o University of California, Berkeley, CA 94720-3840 is published continuously online. Periodical rate postage paid at Berkeley, CA 94704, and additional mailing offices.

ANT peer review and production are managed by EditFLOW ${ }^{\circledR}$ from Mathematical Sciences Publishers.

\section{PUBLISHED BY}

mathematical sciences publishers

nonprofit scientific publishing

http://msp.org/

(C) 2013 Mathematical Sciences Publishers 


\section{Algebra \& Number Theory}

$\begin{array}{lll}\text { Volume } 7 & \text { No. } 2 & 2013\end{array}$

The system of representations of the Weil-Deligne group associated to an abelian variety 243 RUTGER NOOT

Fourier-Jacobi coefficients of Eisenstein series on unitary groups

BEI ZHANG

The phase limit set of a variety

MoUnIR NisSE and FRANK SoTTILE

Base change behavior of the relative canonical sheaf related to higher dimensional moduli

Zsolt PATAKFALVI

Two ways to degenerate the Jacobian are the same

JESSE LEO KASS

Arithmetic motivic Poincaré series of toric varieties

Helena Cobo Pablos and Pedro Daniel González Pérez

Maximal ideals and representations of twisted forms of algebras

Michael LAU and ARTURo PiAnZOLA

Higher Chow groups of varieties with group action 